\title{
A Real-Time Time-Frequency Based Instantaneous Frequency Estimator
}

\author{
Ljubiša Stanković* ${ }^{*}$ Miloš Daković*, Thayananthan Thayaparan**,
}

\begin{abstract}
Commonly used time-frequency representations, like the short-time Fourier transform, the Wigner distribution and the higher order polynomial distributions, estimate the instantaneous frequency at the middle of the time-interval used in the analysis. For real time applications, like for example in radar signal processing, where the target parameters are estimated in the same way as the instantaneous frequency, the delay of a half of the considered interval may be unacceptable. Here, we propose a distribution that inherently estimates the instantaneous frequency at the end of the considered time interval. With a presented procedure for on-line implementation it can outperform other time-frequency representations for real time instantaneous frequency estimation.
\end{abstract}

\section{INTRODUCTION}

Estimation of the instantaneous frequency (IF) is of great importance in many applications. For example, in radar signal processing it produces information about target's range and cross-range position. Many IF estimators are based on the time-frequency representations [1]-[7]. Most commonly used are the short time Fourier transom (STFT), the Wigner distribution (WD) and other quadratic reduced interference distributions, defined by the Cohen class. Higher order spectra and distributions are introduced in order to improve the IF estimation, as well. In most of them the IF estimate is obtained for the mid point of the lag interval. Therefore, the IF estimate is delayed for a half of the lag interval. In radars, for example, it means that the estimate of target data is done with a delay corresponding to the half of lag interval (coherence integration time-CIT). This delay can be significant and unacceptable in many cases. Some efforts have been made in the IF extrapolation, in order to deal with this problem.

In this paper we will present a distribution that inherently estimates the IF value at the ending point of the lag window. The distribution preserves property of the WD that it is fully concentrated if the phase variations of signal are up to the quadratic order. Bias and variance analysis of the proposed IF estimator, in the case of signals with nonlinear IF, is done. Simulations show the efficiency of the presented distribution, when the current instant for the IF estimate is considered as relevant, rather than the one delayed for half of the lag interval. A procedure for reduced crossterms realization of the proposed distribution is described. This procedure can be used in the multicomponent signals analysis. It improves performance in the case of high noise, as well.

${ }^{*}$ Elektrotehnički fakultet, University of Montenegro, 81000 Podgorica, Montenegro.

${ }^{* *} \mathrm{R} \& D$, Ministry of Defence, Ottawa, Canada.

\section{DeFINITION}

In recent research, an interest to the highly concentrated distributions has been again increased. For a frequency modulated signal $x(t)=A \exp (j \varphi(t))$ the model of a fully concentrated distribution, along the IF

$$
T F(t, \omega)=2 \pi A^{2} \delta\left(\omega-\varphi^{\prime}(t)\right)=F T_{\tau}\left[A^{2} e^{j \varphi^{\prime}(t) \tau}\right]
$$

is set again as the basic reference model in sparse timefrequency signal processing (and other recent developments). Assume that the phase function could be considered as quadratic, within the lag time interval. Our goal here is to find the simplest distribution, that is fully concentrated at $\varphi^{\prime}(t)$, at the current instant $t$. In contrast to the other distributions derived in a similar way, here we impose the constraint that only the past signal values are available for calculation. This kind of constraint is especially important in real time applications where an IF estimation, delayed by a half of the lag interval, may not be acceptable. In general, time-frequency distributions are defined as the Fourier transform (FT) of generalized localautocorrelation functions. Here, in the definition of the local auto-correlation function, we use only the current and past instants as

$$
r(t, \tau)=e^{j\left[a_{0} \varphi(t)+a_{1} \varphi(t-\tau / 2)+a_{2} \varphi(t-\tau)\right]}=e^{j \varphi^{\prime}(t) \tau} .
$$

Without loss of generality, a signal normalized in amplitude $A=1$ is used. We also assume that the lag values are of the form $\tau$ and $\tau / 2$, so that they can be sampled with sampling interval corresponding to the WD calculation i.e. with $\Delta t / 2$. Therefore, an interpolation of discrete signal is not required. After an expansion of the phase functions $\varphi(t-\tau / 2)$ and $\varphi(t-\tau)$ into a Taylor series arount $t$, the coefficients should satisfy the following system: $a_{0}+a_{1}+a_{2}=0 ;-a_{1} / 2-a_{2}=1$ and $a_{1} / 8+a_{2} / 2=0$. It produces $a_{0}=3, a_{1}=-4$ and $a_{2}=1$, with

$$
r(t, \tau)=x^{3}(t) x^{* 4}\left(t-\frac{\tau}{2}\right) x(t-\tau) .
$$

A time-frequency representation (TFR) of a continuous signal $x(t)$, then, is

$$
D(t, \omega)=\int_{0}^{\infty} x^{3}(t) x(t-\tau) x^{* 4}\left(t-\frac{\tau}{2}\right) e^{-j \omega \tau} d \tau .
$$

Integration is from 0 to $\infty$ since this distribution is defined to be causal. Notation $x^{* n}(t)$ means the $n$-th power of complex conjugate of a signal. Since the causal from is used, the reprsentation of a signal $x(t)=A \exp (j \varphi(t))$, with $\varphi^{\prime \prime \prime}(t)$ being negligible, is

$$
D(t, \omega)=\pi A^{2} \delta\left(\omega-\varphi^{\prime}(t)\right)+\frac{j 2 \omega}{\omega-\varphi^{\prime}(t)}
$$


with second term, which does not change the nature of distribution begin concentrated at $\omega=\phi^{\prime}(t)$.

Its pseudo form reads

$$
D(t, \omega)=\int_{0}^{h} w(\tau) x^{3}(t) x(t-\tau) x^{* 4}\left(t-\frac{\tau}{2}\right) e^{-j \omega \tau} d \tau .
$$

The past time interval $[t-h, t]$ is used for $D(t, \omega)$ calculation. A lag window is denoted by $w(\tau)$ for $\tau \in[0, h]$. Note that the pseudo WD definition, by using signal values from the same interval, would be of the form

$$
W D\left(t-\frac{h}{2}, \omega\right)=\int_{0}^{h} w(\tau) x\left(t-\frac{h}{2}+\frac{\tau}{2}\right) x^{*}\left(t-\frac{h}{2}-\frac{\tau}{2}\right) e^{-j \omega \tau} d \tau
$$

In order to analyze performance of (6) as an IF estimator, let us consider a single component frequency modulated signal

$$
x(t)=A(t) e^{j \varphi(t)},
$$

with small amplitude changes over the considered time interval, i.e., $A(t-\tau) \cong A(t)$ for $0 \leq \tau \leq h$ and $\varphi(t)$ is a continuous and differentiable function of time. The IF of this signal is

$$
\omega_{i}(t)=\frac{d \varphi(t)}{d t}=\varphi^{\prime}(t),
$$

with the phase in the local auto-correlation function of the form

$$
\begin{aligned}
3 \varphi(t)+\varphi(t-\tau)-4 \varphi\left(t-\frac{\tau}{2}\right) & =\varphi^{\prime}(t) \tau-\frac{1}{12} \varphi^{\prime \prime \prime}(t) \tau^{3}+\ldots \\
& =\varphi^{\prime}(t) \tau+\Delta \varphi(t, \tau) .
\end{aligned}
$$

The IF estimation is based on

$$
D(t, \omega) \cong A^{8}(t) W\left(\omega-\varphi^{\prime}(t)\right) *_{\omega} F T\left\{e^{-j \frac{1}{12} \varphi^{\prime \prime \prime}(t) \tau^{3}+\ldots}\right\} .
$$

where $W(\omega)$ is the FT of $w(\tau)$. Maximal value of $|D(t, \omega)|^{2}$ is reached at $\omega=\varphi^{\prime}(t)$ with a possible bias caused by the third and higher order derivatives of phase. Note that in the WD case the maximal value would be reached at $\omega_{W D}=$ $\varphi^{\prime}(t-h / 2)$.

This distribution has been defined with the aim to be real-time instantaneous frequency estimator, in the first place. It also satisfies some other time-frequency representation properties. It preserves shift in time and frequency. For $y(t)=x\left(t-t_{0}\right) \exp \left(j \omega_{o} t\right), D_{y}(t, \Omega)=D_{x}\left(t-t_{0}, \omega-\omega_{0}\right)$. In addition for a signal $y(t)=x(t) e^{j a t^{2} / 2}, D_{y}(t, \omega)=D_{x}(t, \omega-$ $a t$ ). Time marginal is $\int_{-\infty}^{\infty} D(t, \omega) d \omega / 2 \pi=|x(t)|^{8}$. For a scaled version of the signal $y(t)=\sqrt{|a|} x(a t), a \neq 0$, this distribution reads $D_{y}(t, \omega)=D_{x}(a t, \omega / a)$.The time constraint is satisfied, as well, since $D(t, \omega)=0$, when $x(t)=0$.

\section{Estimator Performance}

A discrete-time form definition of (6), at an instant $t=$ $n T$, reads:

$$
D(t, \omega)=\sum_{k=0}^{N-1} w_{h}(k T) x(t-2 k T) x^{* 4}(t-k T) e^{-j 2 \omega k T} .
$$

The lag independent part $x^{3}(t)$ is omitted, since it will not influence the IF analysis. Consider a noisy signal

$$
x(n T)=s(n T)+\varepsilon(n T)=A e^{j \varphi(n T)}+\varepsilon(n T),
$$

where $\varepsilon(n T)$ is a complex i.i.d. white Gaussian stationary noise with zero mean and variance $\sigma_{\varepsilon}^{2}$, and $\varphi(n T)$ are discrete time samples of the differentiable continuous function $\varphi(t)$, with bounded derivatives.

The IF, at a time instant $t=n T$, is estimated by:

$$
\hat{\omega}(t)=\arg \max _{\omega}|D(t, \omega)|^{2}=\underset{\omega}{\arg \max _{\omega}} F(t, \omega),
$$

where $F(t, \omega)=D(t, \omega) D^{*}(t, \omega)$.

In order to analyze the estimators performance, we will linearize $\partial F(t, \omega) / \partial \omega$ around the stationary point where $\partial F(t, \omega) / \partial \omega_{\mid 0}=0$, with respect to the estimation error $\Delta \hat{\omega}$, noise and higher order phase terms [8]

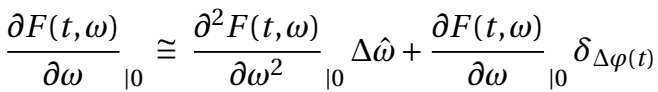

$$
\begin{aligned}
& +\frac{\partial F(t, \omega)}{\partial \omega}{ }_{10} \delta_{\varepsilon_{t}} .
\end{aligned}
$$

Approach to the analysis of this expression is similar to the one applied in the WD case, [8]. However, since the distribution $D(t, \omega)$ is not real-valued, it is not straightforward. Thus, we will provide few basic steps for this derivation in the case of complex-valued distributions.

The first derivative in (15) is calculated as

$$
\frac{\partial F(t, \omega)}{\partial \omega}=2 \operatorname{Re}\left[\frac{\partial D(t, \omega)}{\partial \omega} D^{*}(t, \omega)\right] .
$$

The second derivative $\partial^{2} F(t, \omega) / \partial \omega^{2}$, at the stationary point (with $\varepsilon(n T)=0, \omega=\varphi^{\prime}(t)$ and $\Delta \varphi(t, \tau)=0$, [8]), is obtained in the form,

$$
\frac{\partial^{2} F(t, \omega)}{\partial \omega^{2}}=8 A^{10}\left[-V_{2} V_{0}+V_{1}^{2}\right]
$$

where

$$
V_{i}=\sum_{k=0}^{N-1}(k T)^{i} w_{h}(k T) .
$$

The estimation error follows from (15)

$$
\Delta \hat{\omega}=\frac{\frac{\frac{\partial F(t, \omega)}{\partial \omega} \mid 0}{}{ } \delta_{\varepsilon_{t}}+\frac{\partial F(t, \omega)}{\partial \omega} \mid 0{ }_{\Delta \varphi(n T)}}{8 A^{10}\left[V_{2} V_{0}-V_{1}^{2}\right]} .
$$

The estimation bias is

$$
\text { bias } \Delta \hat{\omega}=\frac{1}{8 A^{10}\left[V_{2} V_{0}-V_{1}^{2}\right]} \frac{\partial F(t, \omega)}{\partial \omega{ }_{10}} \delta_{\Delta \varphi(n T)},
$$

where

$$
\begin{gathered}
\frac{\partial F(t, \omega)}{\partial \omega} \delta_{\Delta \varphi(n T)}= \\
A^{10} \sum_{k=3}^{\infty} \frac{3}{4} \varphi^{(k)}(t) \frac{(-2)^{k}-4(-1)^{k}}{k !}\left(V_{k+1} V_{0}-V_{k} V_{1}\right) \\
\approx A^{10} \frac{\varphi^{\prime \prime \prime}(t)}{2}\left(V_{1} V_{3}-V_{4} V_{0}\right),
\end{gathered}
$$

assuming small $\Delta \varphi(t, k T), \exp (j \Delta \varphi(t, k T)) \cong 1+j \Delta \varphi(t, k T)$, $\varepsilon(n T)=0$ and $\omega=\varphi^{\prime}(t)$. Its final form is

$$
\text { bias } \Delta \hat{\omega}=\frac{V_{1} V_{3}-V_{4} V_{0}}{16\left(V_{2} V_{0}-V_{1}^{2}\right)} \varphi^{\prime \prime \prime}(t) \text {. }
$$

The estimation variance is

$$
\operatorname{var} \Delta \hat{\omega}=\frac{1}{\left(8 A^{10}\left[V_{2} V_{0}-V_{1}^{2}\right]\right)^{2}} \operatorname{var} \frac{\partial F(t, \omega)}{\partial \omega}{ }_{10} \delta_{\varepsilon_{t}},
$$


with

$$
\begin{gathered}
\frac{\partial F(t, \omega)}{\partial \omega}{ }_{10} \delta_{\varepsilon_{t}}= \\
2 \operatorname{Re}\left[\sum_{k=0}^{N-1}(-j 2 k T) w_{h}(k T) s(t-2 k T) s^{* 4}(t-k T) e^{-j 2 \omega k T}\right. \\
\times \sum_{k=0}^{N-1} w_{h}(k T) s^{*}(t-2 k T) s^{4}(t-k T) e^{j 2 \omega k T} \\
-\sum_{k=0}^{N-1}(-j 2 k T) w_{h}(k T) x(t-2 k T) x^{* 4}(t-k T) e^{-j 2 \omega k T} \\
\left.\times \sum_{k=0}^{N-1} w_{h}(k T) x^{*}(t-2 k T) x^{4}(t-k T) e^{j 2 \omega k T}\right] .
\end{gathered}
$$

Having in mind that the variance of the real part of a complex zero mean random variable $X$ can be expressed as $\operatorname{var}[2 \operatorname{Re} X]=E\left[X^{2}+X^{* 2}+2 X X^{*}\right]$, after some direct, but lenghty, calculations, with $\omega=\varphi^{\prime}(t)$ and $\Delta \varphi(t, \tau)=0$, for the stationary point, we obtain

$$
\begin{gathered}
\operatorname{var}\left[\frac{\partial F(t, \omega)}{\partial \omega}{ }_{10} \delta_{\varepsilon_{t}}\right]=2 \sigma_{\varepsilon}^{2} A^{18}\left(48 U_{1} V_{1} V_{0}+68 W_{0} V_{1}^{2}\right. \\
\left.+68 W_{2} V_{0}^{2}-136 W_{1} V_{1} V_{0}+32 U_{0} V_{1}^{2}+16 U_{2} V_{0}^{2}\right)
\end{gathered}
$$

where

$$
\begin{aligned}
W_{i} & =\sum_{k=0}^{N-1}(k T)^{i} w_{h}^{2}(k T) \\
U_{i} & =\sum_{k=0}^{N / 2-1}(k T)^{i} w_{h}(k T) w_{h}(2 k T) .
\end{aligned}
$$

Finally, the estimator variance can be written as

$$
\begin{gathered}
\operatorname{var} \Delta \hat{\omega}=\frac{\sigma_{\varepsilon}^{2}}{A^{2}} \frac{1}{32\left[V_{2} V_{0}-V_{1}^{2}\right]^{2}}\left(12 U_{1} V_{1} V_{0}+17 W_{0} V_{1}^{2}\right. \\
\left.\quad+17 W_{2} V_{0}^{2}-34 W_{1} V_{1} V_{0}+8 U_{0} V_{1}^{2}+4 U_{2} V_{0}^{2}\right) .
\end{gathered}
$$

The theoretical variance is checked against the statistically obtained one, within the example.

For a rectangular window $w_{h}(n T)$, a simple form of the variance follows:

$$
\operatorname{var} \Delta \hat{\omega}=\frac{\sigma_{\varepsilon}^{2}}{A^{2}} \frac{9}{2 T^{2}} \frac{5 N+3}{N(N+1)\left(N^{2}-1\right)} \approx \frac{\sigma_{\varepsilon}^{2}}{A^{2}} \frac{45}{2 T^{2} N^{3}} .
$$

In terms of the window length $h=N T$, the variance is $\operatorname{var} \Delta \hat{\omega} \approx 22.5 \sigma_{\varepsilon}^{2} T /\left(A^{2} h^{3}\right)$. For the WD with the same rectangular window, we have [8], $\operatorname{var} \Delta \hat{\omega}_{W D} \approx 6 \sigma_{\varepsilon}^{2} T /\left(A^{2} h^{3}\right)$, meaning that the presented estimator have a higher variance than the WD, applied to the same signal. Note that the WD provides estimation at the central point of the considered lag interval resulting in estimation delay of $h / 2$ with respect to the current time-instant $t$. Time delay in the WD based estimation can produce significant bias and increase total MSE. Its value is $M S E_{W D}=(\operatorname{bias} \Delta \hat{\omega})_{W D}^{2}+\left(\varphi^{\prime}(t)-\varphi^{\prime}(t-h / 2)\right)^{2}+$ $6 \sigma_{\varepsilon}^{2} T /\left(A^{2} h^{3}\right)$. Thus, the proposed distribution will produce better results, if the variation of the IF within the lag window is such that $\left(\varphi^{\prime}(t)-\varphi^{\prime}(t-h / 2)\right)^{2}>16.5 \sigma_{\varepsilon}^{2} T /\left(A^{2} h^{3}\right)$. For the SNR of $10 d B, A^{2} / \sigma_{\varepsilon}^{2}=10$ and quite small number of samples (producing high estimation variance) $h=16$, $T=1$ and $N=16$ it follows that the proposed IF estimator is still better if $\left|\varphi^{\prime}(t)-\varphi^{\prime}(t-h / 2)\right|>0.006 \omega_{m}$, where $\omega_{m}$ is the maximal frequency. Increasing the number of samples will additionally favor the proposed estimator. Thus, we may conclude if any time variation of the IF is expected the proposed estimator will produce better estimate, at the current time instant $t$, than the $\mathrm{WD}$ for any reasonable window width.

It is also well known that all higher order approaches require high values of SNR for successful estimation. In order to illustrate this effect and propose a method for its reduction, consider a very simplified case of a sinusoid $x(n)$ with a frequency $k_{0}$. Assume that its FT is $X(k)=$ $A \delta\left(k-k_{0}\right)+E_{1} \delta\left(k-k_{1}\right)$ where $E_{1} \delta\left(k-k_{1}\right)$ is a disturbing term. Then, we will have the correct estimate of frequency $k_{0}=\arg \left\{\max _{k}|X(k)|\right\}$ if the disturbing term is lower than the desired signal $A>\max \left\{E_{1}\right\}$. Now consider a higher order distribution, requiring the powers of $x(n)$, for example $x^{2}(n)$. Its FT is $X(k) *_{k} X(k)=A^{2} \delta\left(k-2 k_{0}\right)+E_{1}^{2} \delta\left(k-2 k_{1}\right)+$ $2 A E_{1} \delta\left(k-k_{0}-k_{1}\right)$. It is clear that if $A>\max \left\{E_{1}\right\}$ then $A^{2}>\max \left\{E_{1}^{2}\right\}$, i.e., the first two terms in $X(k) *_{k} X(k)$, representing the auto-terms of signal and disturbing term in convolution, will not influence the estimation, in this sense. However, the cross-term $2 A E_{1} \delta\left(k-k_{0}-k_{1}\right)$ is introduced, as well. For the correct estimation, here we must have $A>\max \left\{2 E_{1}\right\}$. It corresponds to the well known $6[\mathrm{~dB}]$ worsening with each higher order degree. Thus, our idea will be to remove, or reduce the cross-terms, as much as possible (in an ideal case to get Cross-TermsReduced $\left.\left\{X(k) *_{k} X(k)\right\}=A^{2} \delta\left(k-2 k_{0}\right)+E_{1}^{2} \delta\left(k-2 k_{1}\right)\right)$ and to improve the estimation limit for low SNR, keeping higher order representations property of improving concentration for non-stationary signals.

\section{The S-Method BASEd REALIZATION}

Based on (6) we can write

$$
D(t, \omega)=x^{3}(t) S T F T_{x}(t, \omega) *{ }_{\omega} S T F T_{\chi^{4}}(t, 2 \omega)
$$

where $\chi(t)=x^{*}(t-\tau / 2)$ and $\operatorname{STFT}_{\chi^{4}}(t, \omega)=\operatorname{STFT}_{\chi}(t, \omega) *_{\omega}$ $S T F T_{\chi}(t, \omega) *_{\omega} S T F T_{\chi}(t, \omega) *_{\omega} S T F T_{\chi}(t, \omega)$. Let us now assume that components in $S T F T_{\chi}(t, \omega)$ are localized i.e. that a component centered at any $\omega_{0}$ is spread in frequency, but only over a region $\left[\omega_{0}-\omega_{L}, \omega_{0}+\omega_{L}\right]$. Then

$$
\begin{aligned}
& S T F T_{\chi}(t, 2 \omega) *{ }_{\omega} S T F T_{\chi}(t, 2 \omega) \\
= & \frac{1}{\pi} \int_{-\infty}^{\infty} S T F T_{x}(t, \omega+\xi) S T F T_{x}(t, \omega-\xi) d \xi \\
= & \frac{1}{\pi} \int_{-\omega_{L}}^{\omega_{L}} S T F T_{x}(t, \omega+\xi) S T F T_{x}(t, \omega-\xi) d \xi
\end{aligned}
$$

This may be considered as a windowed convolution, corresponding to the S-method, [9]. It will reduce all crossterms. The ross-terms between components being at least $2 \omega_{L}$ apart will be completely removed.

Now we will present discrete form for the proposed distribution (6) realization based on this method, that will reduce the effect of high noise to the proposed distribution and also provide possibility of its direct application to the multicomponent signals.

Let us consider a single time instant $t$ and discrete samples $x_{t}(n)$ of a continuous signal $x(t-\tau)$ sampled along $\tau$. We will assume that a rectangular window function $w(n)$ of length $N$ is used. 
Step 1: Calculate a discrete signal $\chi_{t}(n)$ as samples of $x^{*}(t-\tau / 2)$. Its Fourier transform is $\operatorname{STFT}_{1}(t, k)=F T\left[\chi_{t}(n)\right]$ for $-N / 2 \leq k \leq N / 2-1$.

Step 2: We will now calculate $S T F T_{2}(t, k)=F T\left[\chi_{t}^{2}(n)\right]$ by convolution

$$
\operatorname{STFT}_{2}(t, k)=\sum_{p} S T F T_{1}(t, p) S T F T_{1}(t, k-p)
$$

Let us assume that components in $\operatorname{STFT}_{1}(t, k)$ are localized i.e. that a component centered at any $k_{0}$ is spreaded over the region $\left[k_{0}-L, k_{0}+L\right]$. It means that only frequency range $[k-L, k+L]$ will be used for calculation of $\operatorname{STFT}_{2}(t, k)$ for all $k$ since $k_{0}$ is not known.

The limits on $p$ in (32) are $k_{0}-L \leq p \leq k_{0}+L$ and $k_{0}-L \leq$ $k-p \leq k_{0}+L$. Eliminating unknown $k_{0}$ we get

$$
k / 2-L \leq p \leq k / 2+L
$$

Component in $S T F T_{1}(t, k)$, corresponding to $k_{0}$, is located in $S T F T_{2}(t, k)$ within the range $\left[2 k_{0}-2 L, 2 k_{0}+2 L\right]$.

Step 3: The second convolution is performed in the same manner as in Step 2, to obtain

$$
\begin{aligned}
\operatorname{STFT}_{4}(t, k) & =F T\left[\chi_{t}^{4}(n)\right] \\
& =\sum_{p} S T F T_{2}(t, p) S T F T_{2}(t, k-p) .
\end{aligned}
$$

Similar analysis, as in Step 2, leads to the summation limits $\frac{k}{2}-2 L \leq p \leq \frac{k}{2}+2 L$. Note that component corresponding to $k_{0}$ in $\operatorname{STFT}_{1}(t, k)$ is located in $S T F T_{4}(t, k)$ within the range $\left[4 k_{0}-4 L, 4 k_{0}+4 L\right]$. The convolution $\operatorname{STFT}_{4}(t, k)$ contains $4 N-3$ frequency samples in total.

Step 4: We will now calculate $\operatorname{STFT}_{x}(t, k)=F T\left[x_{t}(n)\right]$. For component corresponding to $k_{0}$ in $\operatorname{STFT}_{1}(t, k)$ the corresponding component in $S T F T_{x}(t, k)$ is located within $\left[-2 k_{0}-4 L,-2 k_{0}+4 L\right]$. It is obvious, since $\operatorname{STFT}_{1}(t, \omega)=$ $F T\left[x^{*}(t-\tau / 2)\right]$ and $\operatorname{STFT}_{x}(t, \omega)=F T[x(t+\tau)]$.

The final distribution, with local calculation, is

$$
D(t, k)=\sum_{p} S T F T_{x}(t, k) S T F T_{4}(t, k-p)
$$

where limits for $p$ are $-k-4 L \leq p \leq-k+4 L$.

Note that we can calculate convolutions in different order and obtain similar results. The signal dependent S-method realization does require any distance between auto-terms. It would here produce a cross-terms free form of the Ddistribution if the components do not overlap in the STFT.

Presented theory is illustrated by an example with a noisy signal $x(n)=\exp \left(j 10 \pi n^{2} / N^{2}\right)+\varepsilon(n)$, where the SNR is changed within $-10 \leq S N R \leq 20, N=256$ and $T=1$. The value of $D(t, k)$ is calculated by definition. Its reduced interference form is calculated by using the proposed procedure with $L=7$. The IF is estimated, with additional fine tuning using the interpolation and displacement technique. The MSE is calculated in 1000 realizations. The same is done for the WD. The MSE in WD is limited by a large bias, being equal to the change of the IF within a half of the lag interval. The proposed calculation procedure significantly improves the estimation for low and moderate SNR. The theoretically obtained MSE (29) for $D(t, k)$ is presented, as well, Fig.1.

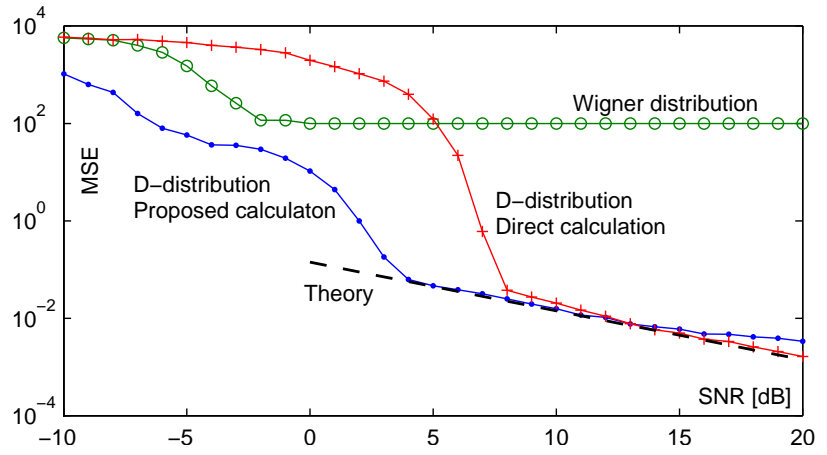

Fig. 1. The estimation MSE, in frequency steps, for a noisy signal: (o) the Wigner distribution, $(+)$ the proposed D distribution, calculated by definition, $(\cdot)$ the proposed $\mathrm{D}$ distribution, calculated by the proposed procedure, (- -) theoretically obtained MSE values.

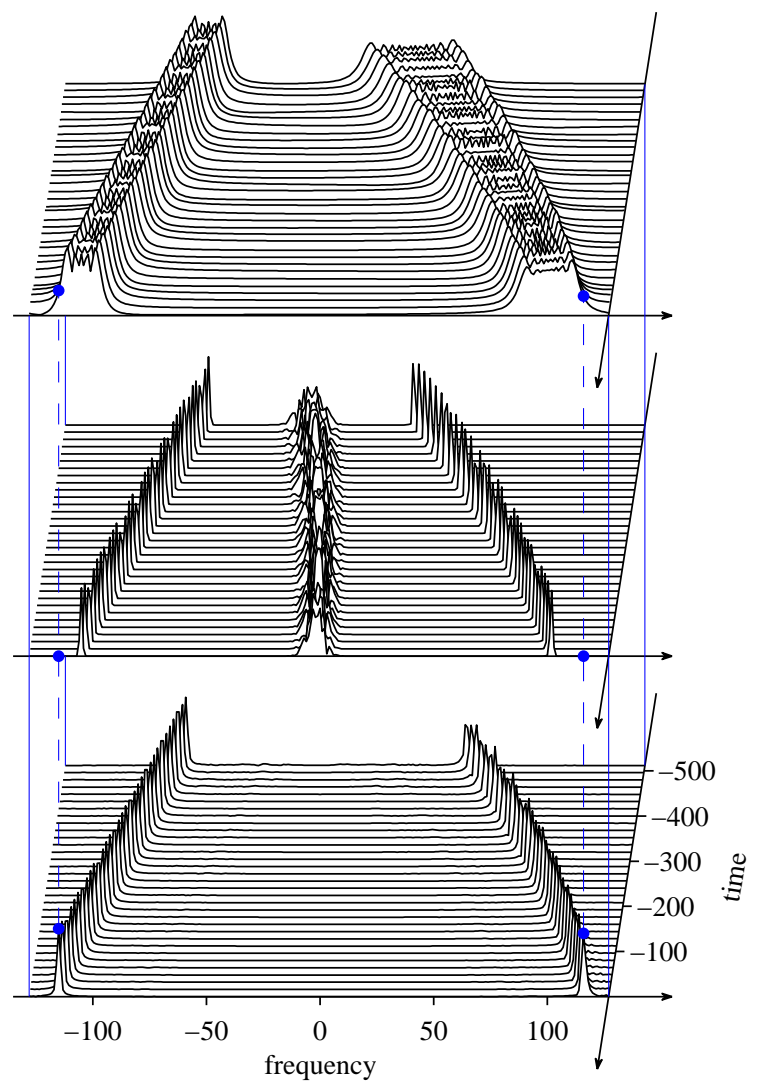

Fig. 2. Time-frequency representation of a two-component signal: The Spectrogram (top), the Wigner distribution (middle) and the D-distribution with proposed cross-terms free (reduced) realization (bottom). Vertical dashed lines indicate true instantaneous frequencies, at the initial time instant, for each component. 

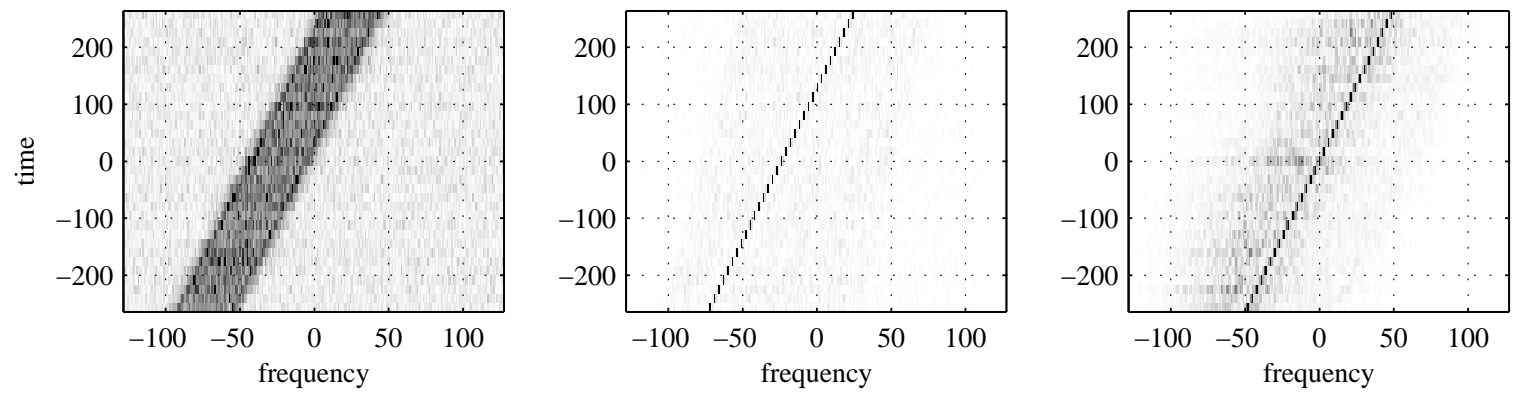

Fig. 3. Time-frequency representation of a mono-component noisy signal: The spectrogram (left), the Wigner distribution (alias free realization), (middle) and the D-distribution with proposed cross-terms free (reduced) realization (right). Signal to noise ratio is $\mathrm{SNR}=5$ [dB].

The STFT can be realized in a recursive (on-line) manner, [9]. Then, with a few multiplications and additions the function $D(t, k)$ is obtained, for each $k$, in a numerically efficient way. It makes real time application of the proposed distribution complete (with possible hardware, online, implementation). In theory, the value of $2 L$ should be equal to the signal component width, but in practice just a few samples around each frequency will significantly improve the concentration. The method will work well in time-frequency representation of multicomponent signals, since the presented calculation procedure removes (reduces) cross-terms.

A realization of the STFT, the WD and the proposed Ddistribution (by definition and by using the S-method based calculation procedure) on a two-component signal

$$
\begin{aligned}
x(n)= & \exp \left(j 230 \pi(n / N)+j 24 \pi(n / N)^{2}-j 10(n / N)^{3}\right) \\
& +\exp \left(-j 230 \pi(n / N)-j 20 \pi(n / N)^{2}\right),
\end{aligned}
$$

with $N=256$ and on a mono-component noisy signal

$$
x(n)=\exp \left(j 48 \pi(n / N)^{2}\right)+\varepsilon(n)
$$

with $S N R=5[\mathrm{~dB}], L=7$ is presented in Fig.2 and in Fig.3. Note that the IF representation in the D-distribution corresponds to the current instant (last available instant in calculation), while the IF in the WD is significantly delayed with respect to this instant, Fig.2. In the spectrogram we can see that the IF, corresponding to the current instant, is at the position of one of the ending frequencies in the wide auto-term. In the Wigner distribution the IF is in the middle of the STFT's auto-term. In the noisy case, the proposed, reduced interference realization (for noise, inspired by the analysis in the last paragraph of Section 3) significantly improves the D-distribution performance. However, it is still a higher order distribution, sensitive to noise. In many applications, when noise is not extremely high, this is the price that is not significant, as compared to the benefit of being able to accurately estimate the current IF. It is not delayed for a half of the lag of window, as in the case of other time-frequency based estimators. The delay of $N / 2$ in the IF estimation can be easily seen in Fig.3.

\section{CONCLUSION}

A distribution that inherently estimated the IF at the last instant of a considered interval is proposed. This distribution, along with a procedure for its reduced interference realization, provides an efficient tool for the IF estimation in real time, when a delay of the half of the lag interval is not acceptable, like for example in radar signal processing, for target localization and identification. The obtained distribution, with the procedure for its calculation, combines good properties of high concentration in higher order distributions and low noise sensitivity of the STFT. It can be directly applied to the multicomponent signals analysis, as well.

\section{REFERENCES}

[1] Z. Hussain and B. Boashash, "Adaptive instantaneous frequency estimation of multicomponent FM signals using quadratic timefrequency distributions," IEEE Trans. SP, vol. 50, pp. 1866-1876, Aug.2002.

[2] P. L. Shui, H. Y. Shang, and Y. B. Zhao, "Instantaneous frequency estimation based on directionally smoothed pseudo-Wigner-Ville distribution bank," IET Radar Sonar Navig., vol. 1, pp. 317-325, August 2007.

[3] M. Ozturk and A. Akan, "Local instantaneous frequency estimation of multi-component signals," Computers and Electrical Engineering, vol. 34, pp. 281-289, July 2008.

[4] B. Barkat and B. Boashash, "Instantaneous frequency estimation of polynomial FM signals using the peak of the PWVD: Statistical performance in the presence of additive Gaussian noise," IEEE Trans. $S P$, vol. 47, pp. 2480-2490, September 1999.

[5] S. Chandra Sekhar, T.V. Sreenivas, "Auditory motivated level-crossing approach to instantaneous frequency estimation," IEEE Trans. SP, vol. 53, no. 4, pp. 1450-1562, April 2005.

[6] E. Sejdic, I. Djurovic, J. Jiang, "Time-frequency feature representation using energy concentration: An overview of recent advances", Digital Signal Processing, 19(1): 153-183 2009.

[7] J. Lerga and V. Sucic, "Nonlinear IF estimation based on the pseudo WVD adapted using the improved sliding pairwise ICI rule," IEEE SP Letters, vol. 16, no. 11, pp. 953-956, 2009.

[8] L. Stankovic and V. Katkovnik, "Instantaneous frequency estimation using the higher order L-Wigner distributions with the data driven order and window length," IEEE Trans. IT, vol. 46, pp. 302-311, Jan.2000.

[9] L. Stanković, "A method for time-frequency analysis," IEEE Trans. SP, vol.42, pp. 225-229, Jan.1994.

[10] L. Stanković, "On the realization of the polynomial wigner-ville distribution for multicomponent signals," IEEE SP Letters, vol. 5, no. 7, pp. 157-159, 1998.

[11] J. Lerga, V. Sucic, B.Boashash, "An Efficient Algorithm for Instantaneous Frequency Estimation of Nonstationary Multicomponent Signals in Low SNR", EURASIP J. on Advances in Signal Processing, ID 725189, vol-2011. 